\title{
Exchange Rates and Asset Prices: heterogeneous agents at work
}

\author{
Giulia Piccillo*
}

January 16, 2009

\begin{abstract}
This paper studies the relationship between exchange rates and asset prices. It takes the novel approach of modeling both the markets in a framework of heterogeneous agents. Investors maximize their profits from the international equity markets by solving a Mean-Variance problem. As a result, agents choose between different combinations of rules in the home and foreign equity market as well as in the foreign exchange market. Given the incomplete information setting, agents check the past profitability of their rules and switch behavior in the effort to maximize their profits.

Due to the heuristics embedded within the model, this simple framework alone is able to create a complex, time-varying dynamics. This dynamics is analyzed for different parameters and conditions. Finally the model is brought to the data, to check the fitness of the predictions on the real world markets.
\end{abstract}

Keywords: Behavioral finance, exchange rates, asset prices.

${ }^{*}$ Center for Economic Studies, Katholieke Universiteit Leuven, Belgium. Telephone: (+32) 01632 6848. E-mail: giulia.piccillo@econ.kuleuven.be. Web page: www.econ.kuleuven.be/giulia.piccillo 


\section{Introduction}

The subject of asset prices and exchange rates has recently received a lot of attention by some of the most renowned economic experts. This is due to two main factors. On the one hand the two time series show similarities typical of financial markets. This makes it possible to use similar statistical tools to study both the markets. On the other hand a full understanding of the economy is essential in order to understand how these markets work. This second factor is what makes the subject attractive for theoretical and macroeconomic contributions as well as for empirical and strictly financial papers. In both cases, a paper studying the relationship between exchange rates and stock prices has to make important economic assumptions. These define the big picture according to the authors and set the tone throughout the paper.

In order to place this paper within the modern literature, it is useful to make a short survey of papers in two separate branches. The first one, in chronological order, is the behavioral finance literature. The second one is the relatively restricted group of contributions that study specifically the relationship between the stock prices and the exchange rates.

Within the wide field of behavioral finance we will go over the heterogeneous agents models, as this is the main assumption in the following paragraphs. The econometric process that is key to the switch between rules has been introduced by Brock and Hommes in 1997 and 1998. Thanks to their econometric paper, the tools were developed to study systems with alternating rules. Frankel and Froot wrote in 1998 a ground breaking paper in this framework.

Finally, De Grauwe and Grimaldi, in 2006 published a book summarizing most of the findings to date and showing new evolutions for the model. Since then, several other contributions have been given, each showing a different aspect of the structure. An example of this, were several fundamental beliefs alternate as well, is the one of De Grawe and Rovira-Kaltwasser, in 2007.

In the area of stock prices and exchange rates, there are several empirical papers. However we would like to just illustrate 3. From the macroeconomic perspective, Kollmann in 2001 develops and solves a DSGE model that includes the capital market. Here for the first time in such a context, the equity flows are studied in conjunction with the exchange rate created by an open economy two country market of the style of (25). The model is solved and the dynamics is studied, however the premises of the DSGE are too wide to study the day to day 
financial series. Nonetheless this work definitely launches some solid foundations to analyze the relationship between stock prices and exchange rates.

The second paper is by Rigobon and Pavlova (2003). They make a general equilibrium model based on productive and demand shocks that influence both the stocks and the exchange rate. Their model is very practical, and the empirical applications all show a good consistency of the data with the model.

Finally, Rey and Hau in 2006 use the exchange rate microstructure intuition, as introduced in (14), to develop a partial equilibrium model with a very wide financial theory section and a strongly supportive empirical application to over 20 countries. All their main findings are found in the data, and to this day it remains the model with the most supportive empirical evidence. They study the exchange rate as the result of order flows for foreign currency from equity trade in an international two country setting. This model, among all the others, catches the importance of flows to and from the two markets.

This contribution focusses on the dynamics created by the interrelation of these markets. For this purpose a theoretical model will be described and analyzed with respect to the series created as well as the empirical relevance of its findings. The next paragraph will describe this model in detail. Paragraph 3 will show a stochastic realization of the model. Then, in section 4, a sensitivity analysis will be undertaken, to study the impact on the time series dynamics, of several of the model parameters. Finally, section 5 will show the similarities between the data created by the model and the real data. The last section will conclude with a brief outlook at future research.

\section{Theory}

The heterogeneous agents model is based on two alternating rules agents use to create expectations. Each rule is continuously revised according to its profitability. If it is profitable agents keep it for the next period, otherwise they switch. While the process is relatively simple, we will see that the dynamics created is fairly complex and it is source of non-linearities as well as heteroscedasticity and time varying behavior.

In our model there are two countries, Home and Foreign. Each country has its own shares, that may be bought by agents from both nations. There are no transaction costs. In this version of the model shares are the only way in which agents may invest. Bonds are not taken into account at this point. This assumption may be relaxed in a following version of this paper, without affecting 
the dynamics between the stocks and the exchange rates.

This setting lays the basic assumptions for three time series to be forecasted. These are the two stock prices (Home and Foreign) and the exchange rate between the two currencies. In order not to make ad hoc assumptions on the evolution of these time series, the framework chosen here is of the most general kind. This approach will allow the researcher to focus on the implications of the irrational expectations element of the model.

\subsection{Stock Prices}

The two stock prices have similar characteristics and share the same news. It will be shown in the next paragraph how even though the same noise will be applied to both markets, their dynamics will be nevertheless different. This is due to the complexity of the behavior of the agents, that is the main driver of the two prices.

So the home stocks are defined by the following specification:

$$
\Delta S_{t+1}=m_{f, t}^{s}[\text { Rule1Forecast }]+m_{c, t}^{s}[\text { Rule } 2 \text { Forecast }]+\varepsilon_{t+1}
$$

where $\Delta S_{t+1}$, the change in tomorrow's stock price, is given by the first rule's forecast weighted by the agents that believe in this rule plus the forecast from the second rule, weighted by the agents believing in this second rule. $\varepsilon$ represents the news, that were not incorporated in either rule.

Given that the two stocks have similar assumptions, it is possible to write the change in Foreign stock prices similarly to the Home stocks. Specifically,

$$
\Delta S_{t+1}^{*}=m_{f, t}^{s *}\left[\text { Rule } 1^{*} \text { Forecast }\right]+m_{c, t}^{s *}\left[\text { Rule } 2^{*} \text { Forecast }\right]+\varepsilon_{t+1}
$$

with $\varepsilon$ being exactly the same news as in the previous equation, and the two rules having also a similar nature (after all we are studying the same series from two different countries). Given the similarity in the assumptions and rules of the two stock markets, from now on we will just describe the home stocks.

Equations 1 and 2 make it clear that expectations are crucial in the evolution of the stock prices. It is now relevant to show the two rules used to make the forecasts. We make assumptions similar to the literature on heterogeneous agents, by assuming that one of the rules will be forward looking, and the other will be backward looking.

The fundamentalist rule, or forward looking, calculates the distance to some value of the stocks believed to be the "fundamental" value, and adjusts this 
distance period after period. More formally:

$$
E_{f, t}\left(\Delta S_{t+1}\right)=-\eta\left(S_{t}-\bar{S}_{t}\right)
$$

The fundamental price of the home stock price will be following a random walk similar to:

$$
\bar{S}_{t+1}=\bar{S}_{t}+\epsilon_{t}
$$

where the noise $\epsilon$ is different for the two stocks, since it includes the information on the national stock itself ${ }^{1}$.

The backward looking rule is, still in tune with the majority of the literature in this field, referred to as a chartist rule, where the relevant information is given by all the past returns, weighted by a discount factor. Formally:

$$
E_{c, t}\left(\Delta S_{t+1}\right)=\beta \sum_{k=1}^{T} \rho(1-r h o)^{k} \Delta\left(S_{t-k}\right)
$$

However a key feature of the system is that the agents are not static, but keep evaluating their returns and check if their rule is still the most profitable one. So if the rule is good they can keep it, otherwise they will switch over to the other rule, in the hope that it will yield them higher returns. Following this idea and applying the fitness criterion based on discrete choice theory, as shown by Brock and Hommes (6), (7), we can calculate the fraction of population that uses each of the rules as a variable of the (risk adjusted) profitability of the same rule. Formally:

$$
m_{f, t}=\frac{\exp \theta \pi_{f, t}^{\prime}}{\exp \theta \pi_{f, t}^{\prime}+\exp \theta \pi_{c, t}^{\prime}} ; \quad m_{c, t}=\frac{\exp \theta \pi_{c, t}^{\prime}}{\exp \theta \pi_{f, t}^{\prime}+\exp \theta \pi_{c, t}^{\prime}}
$$

Obviously in this case $m_{f, t}$ and $m_{c, t}$ are the fractions of population that at a given time are following the two rules, and therefore they add up to 1 at all times. Furthermore the variables $\pi_{f, t}^{\prime}$ and $\pi_{c, t}^{\prime}$ are the risk adjusted profits realized through the use of each of the rules. For example we could think of them as $\pi_{f, t}^{\prime}=\pi_{f, t}-\rho \sigma_{f, t}^{2}$ and $\pi_{c, t}^{\prime}=\pi_{c, t}-\rho \sigma_{c, t}^{2}$ where the symbol $\pi$ represents the profits made by each of the rules, and the $\sigma^{2}$ are a measure of the risk that the rules involve. For this purpose forecast errors could solve the information quest. Finally $\rho$ is a coefficient of risk aversion.

\footnotetext{
${ }^{1}$ If the noise component $\epsilon$ was equal between the two stocks, then we would have foreign stocks moving similarly all over the world, at least in their levels and directions, while it is clearly not what we observe. Therefore this general assumption is the most conservative we can make in order not to include any useless information in our model.
} 
This system of equations shows how the agents revise their decision making rules as one of the two rules becomes more attractive (read profitable). An important factor in this picture is how quick the agents are to revise and eventually correct their rules, concept that is inserted in the model through the parameter $\theta$. For an increasing $\theta$ all agents revise their rules quickly and therefore a higher amount of population follows the most profitable rule at every time $t$. When the agents are insensitive to the profitability of their rules a good starting point could be a 0.5 fraction of the population using each rule.

The model's frame is built, and now the main concept left to formalize is how to calculate the profits $\pi_{i, t}^{\prime}$ for every rule $i$. To begin with, we should define the profits non adjusted for the risk, as the single period returns from 1 unit of local currency invested into a foreign asset:

$$
\begin{aligned}
& \qquad \pi_{i, t}=\Delta S_{t} \operatorname{sgn}\left[E_{t-1}^{i}\left(S_{t}\right)-S_{t-1}\right] \\
& \text { with } \operatorname{sgn}(x)=\left\{\begin{array}{ll}
1, & \text { for } x>0 \\
0, & \text { for } x=0 \\
-1 & \text { for } x<0
\end{array} \text { and } i=c, f\right.
\end{aligned}
$$

Therefore the profit for every rule goes up whenever the forecast is right, and goes down whenever the forecast is far from being correct. In order to calculate the risk associated with every rule we are going to look at the most general case in which it is the forecast error of the previous periods weighted by a discount factor. Therefore, formally:

$$
\sigma_{i, t}^{2}=\sum_{k=1}^{T} \rho(1-r h o)^{k}\left(E_{t-1-k}^{i}\left(S_{t-k}\right)-S_{t-k}\right)^{2}
$$

Having defined all the variables of the system it is possible to describe the market forecast as a weighted average of the two groups' forecasts, as we did in Equation 1. Only, this time Rules 1 and 2 will be written out as in the following equation:

$$
E_{t} \Delta S_{t+1}=m_{f, t}\left[-\eta\left(S_{t}-S_{t}^{*}\right)\right]+m_{c, t}\left[\beta \Delta S_{t}\right]
$$

This being the forecast, the actual change of the exchange rate market, given the described model, is formally written as:

$$
\Delta S_{t+1}=m_{f, t}\left[-\eta\left(S_{t}-S_{t}^{*}\right)\right]+m_{c, t}\left[\beta \Delta S_{t}\right]+\varepsilon_{t+1}
$$

Therefore the market is governed by this dynamics and by a white noise shock occurring in time $t+1$. The foreign stock is going to be following the same 
assumptions. This framework, completed by the same noise in both markets (but different evolution of the fundamental values) gives life to several different dynamics. More in detail, the two stock prices will be two realizations of similar dynamics. Depending on the specification itself, and on the particular solution to the problem, they may be very similar or very different, just like it happens in reality. In this field it is enough to compare on the one hand the SP500 in the US with the FTSE100 for UK, to see two markets with very similar dynamics. On the other hand the same SP500 Index is very different in its evolution, for example, from the NIKKEI Index for the Japanese market. This model may replicate exactly this relationship, depending on which numerical solution we look at.

\subsection{Exchange Rate}

The exchange rate in this model has similar assumptions to the stock prices. Also in this case, the exchange rate value is going to be given by the weighted average of the expectations, plus a noise. However this time agents have more information on what to base their fundamental value on. This comes from the fact that exchange rate is affected by the flow of currencies that go into investing in either of the stock prices. So the expectations of the stock prices directly affect the fundamental exchange rate.

Obviously whoever believes in the chartist rule, will take the exchange rate series to be its own auto-regressive process, with no pegging onto a theoretical pre-set value. So the equations for the fundamental expectation of the exchange rate will be the following:

$$
\begin{aligned}
& E_{f, t}\left(\Delta e_{t+1}\right)=-\eta\left(e_{t}-\bar{e}_{t}\right) \\
& \bar{e}_{t+1}=\bar{e}_{t}+\vartheta\left(R_{S}-R_{S^{*}}\right)
\end{aligned}
$$

with $R_{S}-R_{S^{*}}$ being the difference between the expected returns on the two stocks. This means that the fundamental value of the exchange rate is ties into the expectations of the prices of the two stocks. It makes sense since, in the agents minds, if there is a high difference in the two stocks, the one that gains more relative return will also attract more capital. This will cause a stronger expected currency, in the expected stronger national stock return. 
The chartist expectation is still defined as above:

$$
E_{c, t}\left(\Delta e_{t+1}\right)=\beta_{e} \sum_{k=1}^{T} \rho(1-r h o)^{k} \Delta\left(e_{t-k}\right)
$$

The series of the exchange rate evolves from the expectations as we have seen for the stock prices. Formally:

$$
\Delta e_{t+1}=m_{f, t}\left[-\eta\left(e_{t}-\bar{e}_{t}\right)\right]+m_{c, t}\left[\beta_{e} \Delta e_{t}\right]+\varepsilon_{e, t+1}
$$

This concludes the description of the model used in order to replicate the dynamics of the stock prices and the exchange rates. As shown this theory is based on very intuitive and consistent assumptions. These cause a very complex dynamics, that will be analyzed in a few of its realizations in the next section. Indeed the major trait of this model is that it creates a non-linear dynamics, that is impossible to solve into a general solution, except for making more constrictive assumptions, that would restrict the model. However we feel that this is exactly its most valuable asset. The flexibility of this partial equilibrium model, and its adaptability to include and generate most statistical characteristics found in real time series from the major stocks and exchange rates of the world. This characteristics are the focus of the next section.

\section{Stochastic Simulation}

This section shows the behavior of the series described above. In the following case we set the initial values of the stock prices both to be equal to 20 . Their fundamental values are also set in the beginning to be equal to 20 . The exchange rate is equal to 1 , both in the initial value, and in the first fundamental value. Other parameters are: $\beta=0.9, \beta_{e}=0.9, \psi=0.2, \psi_{e}=0.2, \eta=0.5, \gamma=10$, $\gamma_{e}=700, \rho=0.5, \vartheta=2$. The simulation is shown in Figure 1. The figure shows in yellow the fundamental and actual value of the home stock price. The fundamental value is a ticker line, while the actual value is a thinner line. In green is the foreign stock price and its fundamental. Finally the exchange rate is in red, and its fundamental value is in cyan. The picture shows how for most of the iterations the two stock prices follow closely the fundamental value, while in the end the home stock gets in a bubble, or an absolute predominance of the chartist believers over the fundamentalists. Figure 2 shows the same scenario, with shades on the areas where the weight of the chartists in the series is close to 1 for a relevant amount of time in a row. So the first shaded area shows the exchange rate bubble, which may be easily seen from the cyan and red lines 


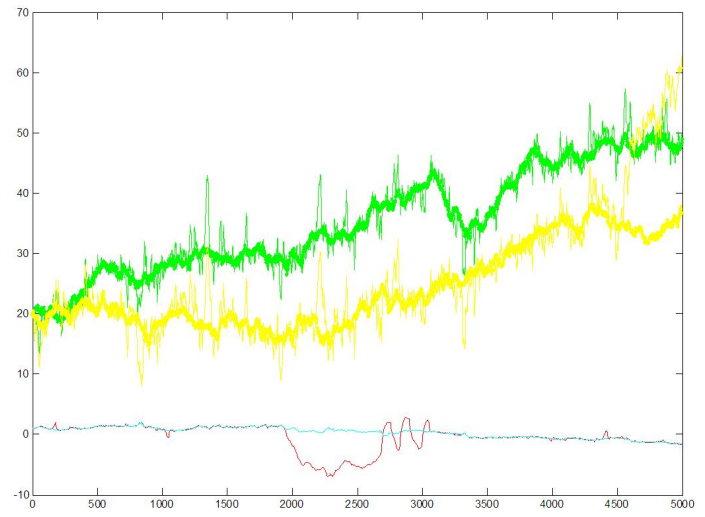

Figure 1: Stochastic simulation with initial conditions of stock prices being equal.

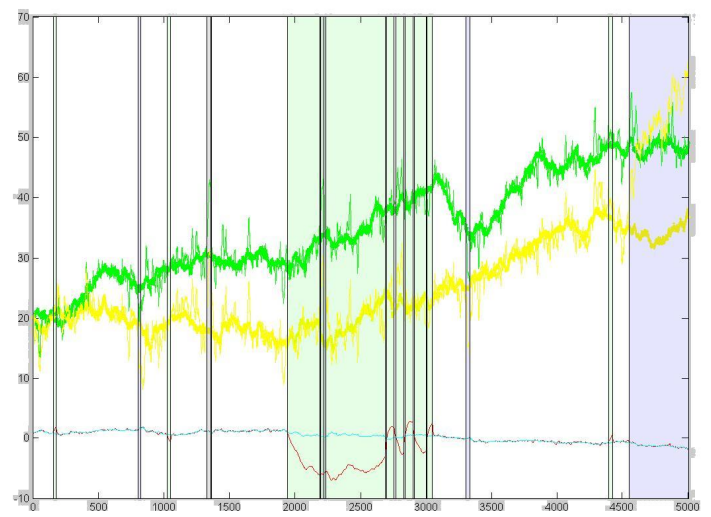

Figure 2: Stochastic simulation with shades on weights of chartists equal to 1 . 


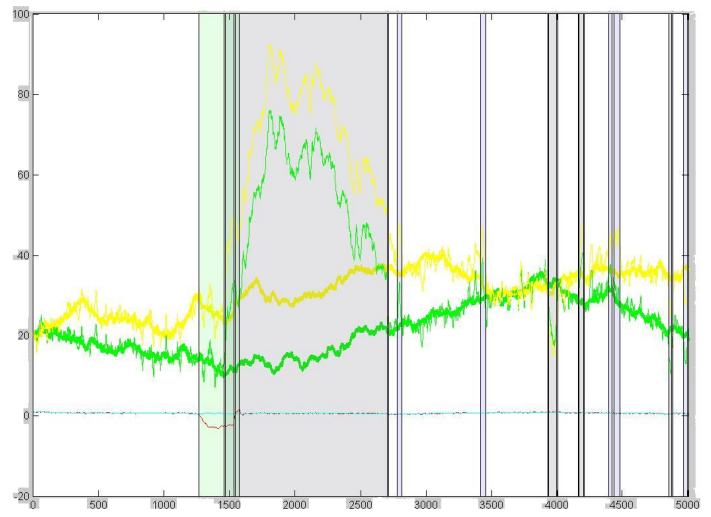

Figure 3: Stochastic simulation with shades on weights of chartists equal to 1 .

diverging. The shaded area on the last part of the graph emphasizes how the home bubble occurs as the weight of chartists is close to 1, and no agent is believing in the fundamentalist rule anymore.

Another scenario is being shown in Figure 3. Some parameters have been modified, to make it more likely for stock prices to create bubble, and less for the exchange rate. This means, as will be shown in the next session, that $\gamma=16, \gamma_{e}=500$. Also, the impact on the exchange rate fundamental rate by the expectations on the stock prices is much lower now, or $\vartheta=0.5$. This may be seen from the flatness of the fundamental exchange rate line, in cyan. This last change decreases the variance of the exchange rate. However this variance is still higher than it would be expected by just looking at the changes in the fundamental value.

Another factor that makes this figure interesting for our study is the fact that the two stock prices have a similar bubble. This means that the common news have been able to trigger a world bubble. From the previous picture we know that this does not always happen. However this model allows also for same time bubbles. This could be compared to the technology bubble of the 1990s, for example, when the stocks were full of that famous "irrational exuberance", and crushed within a short time from each other, in the second half of the decade.

Figure 4 also shows a similar scenario in that bubbles take control of the system in the second half of the sample period. Here the difference between the 


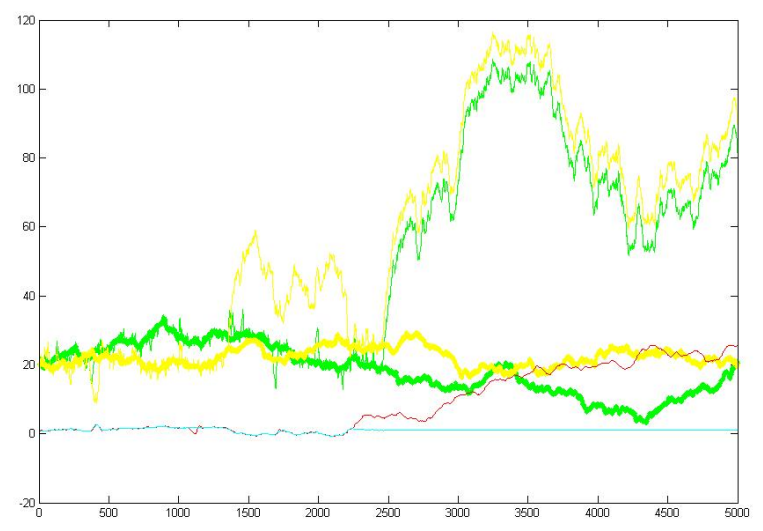

Figure 4: Bubbles in all markets

chartist belief and the fundamentalist is so far that bubbles have a strong pressure to continue. Also the exchange rate shows a larger than average bubble. This is due to the fact that the difference between the two stocks is always constant, making the exchange rate fundamental value stable. In an environment with noise this creates the conditions for a bubble to be developed, because the chartist can better account for the noise. As stated before, these are just a few realizations of the system. To study them all is obviously impossible. However it is interesting (and feasible) to look at the characteristics of the realizations as the parameters change. Furthermore we are going to analyze common statistical traits of the system, and how they compare to the real data.

\subsection{Sensitivity to initial conditions in a stochastic setting}

One of the properties of this system is that it has a long memory, so even small changes in initial conditions may eventually create divergencies in the series. Figure 5 shows two stocks series that have two different initial condition: 10 and 20. Despite the fact that they follow the same fundamental value, it is possible to see how the bubbles occur at different times. This is because of the memory shown by the system. This is a property of complex non-linear systems. This is indeed the property that creates the biggest forecasting problems. This is why, even if it is possible to predict short term series, in the long run forecasts become harder. 


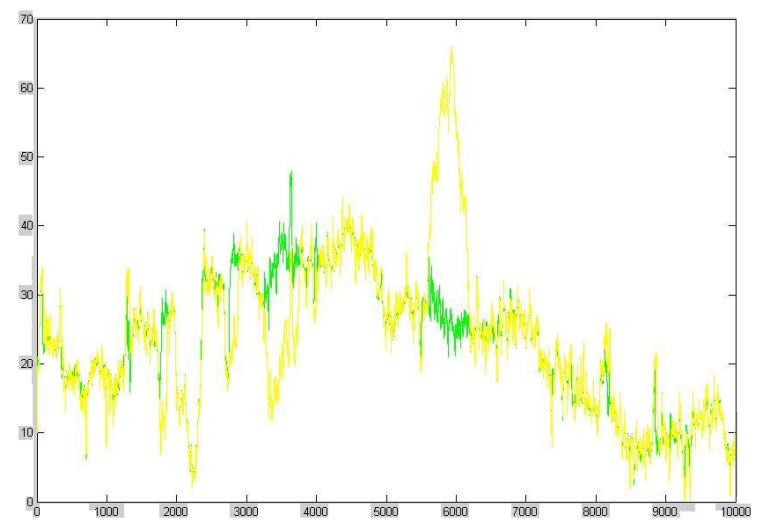

Figure 5: Stocks: sensitivity to initial conditions in a stochastic environment

\subsection{Time series properties}

The parameters of the system influence the outcome of the simulation. So we have the value of $\gamma$ accounting for the amount of times the agents react to the relative returns. This means that a high $\gamma$ allows agents to be constantly revising their rules, which leads to more unified agents. Also, when this parameter is high, we see a higher raise of bubbles. This is because when they are following a fundamental value, there is still some noise. When this is not accounted for by the fundamental value, then agents are quick to unify under the chartist rule and create a bubble. this is what we have showed above.

Moreover the two stock series modeled above have, as mentioned in the theory, the same news from period to period. The only thing that differs is the fundamental value. However the two stocks are always shown to be definitely differen from each other. This means that even with no difference in actual news, but only with different believes on the fundamental value of the series, the dynamics always changes radically. This gives space to papers such as the one by De Grauwe and Rovira-Kaltwasser, which study the heterogeneous agents problem with two variations of the fundamental belief (Optimistic and pessimistic fundamental agents on one side, and the chartists on the other). 


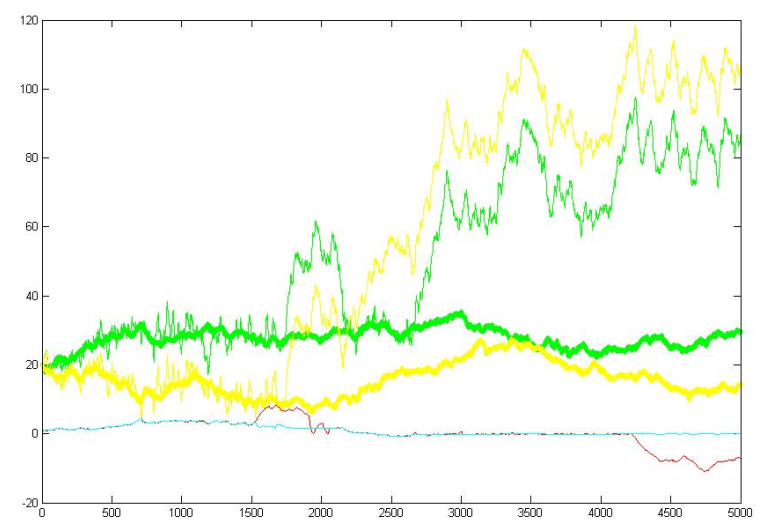

Figure 6: Graph from time series studied

\begin{tabular}{|r|cccc|}
\hline \hline Variable & Coefficient & Std. Error & t-Statistic & Prob. \\
\hline DSF & -0.029 & 0.004 & -7.207 & 0.00 \\
DSH & 0.026 & 0.004 & 6.687 & 0.00 \\
C & -0.001 & 0.000 & -2.850 & 0.00 \\
\hline \hline
\end{tabular}

Table 1: Coefficients of the regression on the exchange rate from simulation

\section{Empirical comparison}

This is section is going to show the similarities (and differences) between the simulated model and the real data. First of all we are going to show the graph of a typical realization. Figure 6 has all the properties identified above. We checked all the series for unit roots. The results were uniform across the series. All of them are integrated of order 1 . Secondly we regress both stocks on the exchange rate series, to verify whether they are significant and consistent with the real data.

Tables 1 and 2 show the results of the regression for both data. As an example of real data we have chosen one of the most studied markets, namely the dollar pound exchange rate. The stock indexes are the S\&P500 for the US and the FTSE100 for the United Kingdom. The period is from 1973 through 2006 on a monthly basis. Figure 8 shows the dynamics of the markets and how they visually look similar in variance and behavior to the simulated data reported 


\begin{tabular}{|r|cccc|}
\hline \hline Variable & Coefficient & Std. Error & t-Statistic & Prob. \\
\hline DFTSE & 0.270 & 0.054 & 4.957 & 0.00 \\
DSP & -0.185 & 0.058 & -3.144 & 0.00 \\
C & -0.001 & 0.001 & -0.881 & 0.38 \\
\hline
\end{tabular}

Table 2: Coefficients of the regression on the dollar-pound exchange rate

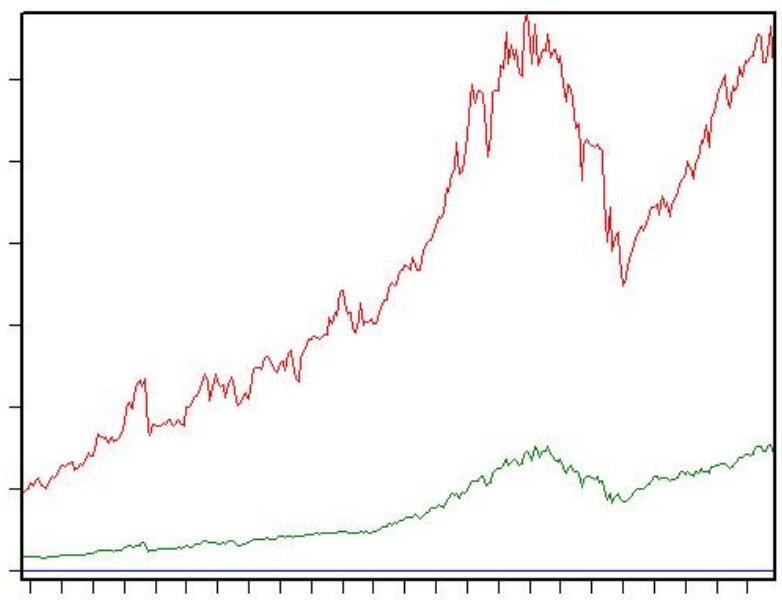

Figure 7: Graph from time series studied

above. Looking at the table it is evident how in both the simulated and the real data the stock returns are very significant in explaining the exchange rate returns. This means that the study at hand is important in the understanding of both the markets.

Finally, the last two considerations concerning the relationship between the two markets. The first one is that the residuals of the two regressions reject the normality assumption both in the data and in the model. The second one is that, as mentioned earlier, the variance of the exchange rate is consistently lower than the one of both stock prices. This is very important to the structuring of the model, since the exchange rate is influenced by the stock prices, but not all the variance is carried over. Furthermore the exchange rate system itself may create endogenous bubbles. Such bubbles are not necessarily tied into the stock price dynamics.

Finally another manner of comparing the data to the theory is to check 
the statistical properties of the single series. We can confirm all the properties shown in (9) and (10), such has high kurtosis, in both exchange rates and stock prices and heteroskedasticity.

\section{Conclusion}

This paper has shown a model of exchange rates and stock prices based on heterogeneous expectations. The model is structured in such a way that most of the dynamics origins from the behavioral finance aspect. This is to emphasize the complexity of the simplest model.

The system was studied and the properties of the time series have been highlighted. Furthermore the model was brought to the data, and its properties compared to the real markets. The traits that were most emphasized were the statistical properties of the single series and the relationship between the stocks and the exchange rates.

All the aspects analyzed have shown similarities rather than differences. However further space for research is needed in finding specific measures to verify the extent of the similarity between the real and the simulated dynamics. This is a key concept since a future addition to this research will be to estimate the model with real data.

Moreover, this work takes into account only a direct relationship between the stocks and the exchange rate. A more structural economic model involving macroeconomic variables is also part of the future developments.

In conclusion it is natural that all of the future research possibilities would not be possible if this simple framework was found to be flawed for this analysis. This is, in short, the role of this paper. We have analyzed a new approach that creates a complex and consistent with data dynamics in the most general of economic assumptions. 


\section{References}

[1] Issam S.A. Abdalla and Victor Murinde. Exchange rate and stock price interactions in emerging financial markets: evidence on india, korea, pakistan and the philippines. Applied financial economics, 7:25-35, 1997.

[2] Patricia Alvarez-Plata and Mechthild Schrooten. The argentinean currency crisis: A markov-switching model estimation. German Institute for economic research, Berlin, Discussion paper 348, May 2003.

[3] Torben G. Andersen, Tim Bollerslev, Francis X. Diebold, and Clara Vega. Real-time price discovery in stock, bond, and foreign exchange markets. National Bureau of economic research, May 2005.

[4] K. N. Badhani. Dynamic reloationship among stock prices, exchnage rate, and net fii: Investment flow in india. 2005.

[5] Bhattacharya Basabi and Mukherjee Jaydeep. Causal relationship between stock market and exchange rate, foreign exchange reserves and value of trade balance: A case study for india. 2001.

[6] W. Brock and C. Hommes. A rational route to randomness. Econometrica, 65:1059-1095, 1997.

[7] W. Brock and C. Hommes. Heterogeneous beliefs and routes to chaos in a simple asset pricing model. Journal of economic dynamics and control, 22:1235-1274, 1998 .

[8] Brad Comincioli. The stock market as a leading indicator: An application of granger causality. The University Avenue Undergraduate Journal of Economics, 1996.

[9] Paul De Grauwe and Marianna Grimaldi. The Exchange Rate in a Behavioral Finance Framework. Princeton University press, 2006.

[10] Paul De Grauwe and Pablo Rovira-Kaltwasser. Modeling optimism and pessimism in the foreign exchange market. CESifo working Paper No. 1962, 2007.

[11] Michael B Devereux and Alan Sutherland. Solving for country portfolios in open economy macro models. 2007. 
[12] Desislava Dimitrova. The relationship between exchange rates and stock prices: Studied in a multivariate model. Issues in Political Economy, 14, August 2005.

[13] C. Engel and J. D. Hamilton. Long swings in the dollar:are they in the data and do the markets know it? American Economic Review, 80:689-713, 1990 .

[14] Martin Evans and Richard Lyons. Order flow and exchange rate dynamics. Journal of Political Economy, Vol. 110, 2002.

[15] J. Hamilton. A new approach to the economic analysis of nonstationary time series and the business cycle. Econometrica, 57(2):357-384, 1989.

[16] Fumio Hayashi. Econometrics. Princeton University Press, 2000.

[17] Soren Johansen. Estimation and hypothesis testing of cointegration vectors in gaussian vector autoregressive models. Econometrica, 59, No. 6:15511580, November 1991.

[18] C.E. Jorion. The exchange rate exposure of us multinationals. Journal of Business, 63 (3), 1990.

[19] Saadet Kasman. The relationship between exchange rates and stock prices: A causality analysis. 2003.

[20] Robert Kollmann. Explaining international comovements of output and asset returns: The role of money and nomical rigidities. Journal of Economic Dynamics and Control, Vol. 25, 2001.

[21] J. P. LeSage. Econometrics toolbox.

[22] Mark and Nelson. International Macroeconomics and finance. Blackwell.

[23] Naeem Muhammad and Abdul Rasheed. Stock prices and exchange rates: Are they related? evidence from south asian countries. 2001.

[24] Jesus Munoz and P. Nicholas Snowden. Floating without flotations- the exchange rate and the mexican stock market: 1995-2001. Journal of International Development, 18:299-318, 2006.

[25] Maurice Obstfeld and Kenneth Rogoff. Exchange rate dyncamics redux. Journal of POlitical Economy, Vol. 103, N. 3, 1995. 
[26] Carol L. Osler. Currency orders and exchange rate dynamics: An explanation for the predictive success oftechnical analysis. The Journal of Finance, October 2003 .

[27] Edoardo Otranto and Giampiero M. Gallo. A nonparametric bayesian approach to detect the number of regimes in markov switching models. Econometric Reviews, 21, No. 4:477-496, 2002.

[28] Anna Pavlova and Roberto Rigobon. Asset prices and exchange rates. Review of Financial Studies, 2007.

[29] Bala Ramasamy and C.H. Matthew Yeung. The causality between stock returns and exchange rates: revisited. Blackwell Publishing Ltd/University of Adelaide and Flinders University, 2005.

[30] Christopher A. Sims and Tao Zha. Mcmc method for markov mixture simultaneous-equation models: a note. 2004.

[31] Christopher A. Sims and Tao Zha. Were there regime switches is US monetary policy? 2006.

[32] C Tille and E van Wincoop. International capital flows. 2006.

[33] Sheng-Yung Yang and Shuh-Chyi Doong. Price and volatility spillovers between stock prices and exchange rates: Empirical evidence from the g-7 countries. International Journal of Business and Economics, 3 (2):139-153, 2004. 